\title{
DISFONIA ORGANOFUNCIONAL E QUEIXAS DE DISTÚRBIOS ALÉRGICOS E/OU DIGESTIVOS
}

\section{Organic-functional dysphonia and complains off allergic and/or digestive disturbance}

\author{
Carla Aparecida Cielo (1), Leila Susana Finger (2), Geise Roman-Niehues (3), \\ Vanessa Panda Deuschle ${ }^{(4)}$, Márcia do Amaral Siqueira ${ }^{(5)}$
}

\begin{abstract}
RESUMO
Objetivo: verificar a ocorrência de queixas de distúrbios alérgicos e/ou digestivos em pacientes com disfonia organofuncional, atendidos no Setor de Voz de uma clínica-escola de Fonoaudiologia. Métodos: este estudo caracteriza-se como estudo de série, retrospectivo e transversal. Realizou-se uma análise de 113 prontuários de pacientes com disfonia organofuncional que apresentavam queixas de distúrbios alérgicos e/ou digestivos, na faixa etária de cinco a 76 anos, sendo 41 do sexo masculino e 72 do feminino. O procedimento estatístico utilizado foi o Teste de Hipótese para Duas Proporções. Resultados: houve significante frequência de queixas de distúrbios alérgicos e digestivos associados nos pacientes dos disfonia organofuncional; entre as queixas de distúrbios alérgicos, de distúrbios digestivos, e de ambas associadas, as queixas de distúrbios alérgicos foram significantemente mais frequentes nos pacientes com disfonia organofuncional. Conclusão: existe uma relação entre as queixas de distúrbios alérgicos e/ou digestivos e a disfonia, uma vez que esses são co-fatores para o estabelecimento da disfonia e da lesão laríngea. A alta freqüência de queixas de distúrbios alérgicos nos indivíduos com disfonia organofuncional, no presente estudo, pode ter relação com o clima do sul do Brasil.
\end{abstract}

DESCRITORES: Distúrbios da Voz; Voz; Refluxo Gastroesofágico; Rinite

\section{INTRODUÇÃO}

A voz é um instrumento de comunicação utilizado pelo ser humano com o intuito de transmitir informações e revelar tanto características biológi-

(1) Fonoaudióloga; Professora Doutora do Programa de PósGraduação em Distúrbios da Comunicação Humana da Universidade Federal de Santa Maria, UFSM, Santa Maria, RS; Doutora em Linguística Aplicada pela Pontifícia Universidade Católica do Rio Grande do Sul.

(2) Fonoaudióloga; Bolsista Capes; Mestranda em Distúrbios da Comunicação Humana pela Universidade Federal de Santa Maria.

(3) Fonoaudióloga Clínica; Mestranda em Distúrbios da Comunicação Humana pela Universidade Federal de Santa Maria.

(4) Fonoaudióloga Clínica; Mestranda em Distúrbios da Comunicação Humana pela Universidade Federal de Santa Maria.

(5) Fonoaudióloga Clínica; Mestre em Distúrbios da Comunicação Humana pela Universidade Federal de Santa Maria.

Conflito de interesse: inexistente cas quanto psicológicas. Muitas vezes, a voz pode informar as condições de saúde, de sexo, de idade, do estado emocional, e até de traços da personalidade de cada indivíduo ${ }^{1,2}$.

Entende-se como disfonia um distúrbio da comunicação, representado por qualquer dificuldade na emissão vocal que impeça a voz de cumprir seu papel básico de transmissão da mensagem verbal e emocional de um indivíduo ${ }^{1-3}$. "Uma desordem vocal existe quando a qualidade, pitch e loudness de uma pessoa diferem dos seus pares em idade, gênero, cultura e localização geográfica. Quando as propriedades perceptivas da voz são tão desviantes que chamam atenção para o falante, uma desordem vocal pode estar presente" 4 .

Disfonias organofuncionais são alterações vocais que acompanham lesões benignas, decorrentes de comportamento vocal alterado ou inadequado 1,5,6, muitas vezes ocorrendo ou sendo agravadas pela presença de fatores orgânicos como os distúrbios alérgicos e ou digestivos ${ }^{7}$. Existem diversos 
estudos relatando a importância de adequadas condições de saúde geral para um bom desempenho da voz ${ }^{8}$, portanto não se pode atribuir uma alteração vocal somente a fatores funcionais, visto que existe uma série de fatores coadjuvantes no processo de estabelecimento das disfonias ${ }^{9}$.

Distúrbios do aparelho digestivo, como dor e dificuldade à deglutição, engasgo, acidez, refluxo gastresofágico ${ }^{10}$, gastrite, digestão lenta e prisão de ventre, dentre outros, prejudicam o processo de fonação de diversas formas, como ao impedir o livre movimento do diafragma, e a aspiração de secreções e alterações secretórias e vasomotoras por estímulo vagal ${ }^{11}$. De 10 a $50 \%$ dos pacientes com queixa de alterações laríngeas têm como causa subjacente o refluxo gastresofágico ${ }^{12}$. O refluxo laringofaríngeo se refere ao refluxo gastresofágico que alcança estruturas superiores ao nível do esfíncter esofágico superior, e isso implica em uma série de alterações otorrinolaringológicas, incluindo laringite posterior crônica, nódulos vocais, laringoespasmo, edema de Reinke, úlceras, granuloma, e sensação de bola na garganta ou globus pharyngeus ${ }^{12-14}$.

Alergias são respostas anormais e especificas a substâncias que normalmente afetam negativamente as pessoas. Indivíduos com alergias de vias aéreas superiores ou inferiores possuem maior tendência a apresentar alterações vocais. Bronquite, asma, rinite, sinusite, laringite, e faringite podem provocar edema nas mucosas do trato respiratório, incluindo as pregas vocais ${ }^{6,15,16}$. Os distúrbios alérgicos constituem a causa mais frequente do prolongamento da terapia fonoaudiológica ${ }^{5}$. Os quadros alérgicos são mais incapacitantes para o usuário da voz profissional do que para outros indivíduos, em decorrência do que causam à mucosa que reveste o trato vocal. É relevante ressaltar a rinite alérgica como importante comprometedor das condições do trato vocal, pois envolve nariz, ouvidos, garganta, o que, no mínimo, compromete a boa projeção da vOZ $^{6}$.

Desta forma, o tratamento das disfonias requer a atuação conjunta e complementar entre o médico otorrinolaringologista, que trata as afecções laríngeas e o seu curso, e entre o fonoaudiólogo, que orienta e trata os comportamentos de uso da voz para que alterações teciduais por uso vocal incorreto não surjam.

No caso de alterações teciduais laríngeas estarem instaladas, o fonoaudiólogo trabalha para que não se agravem, ou para que até possam ser reabsorvidas, como em casos de nódulos vocais.

Com base no exposto, o objetivo do presente estudo foi verificar a ocorrência de queixas de distúrbios alérgicos e/ou digestivos em pacientes com disfonia organofuncional, atendidos no Setor de
Voz de uma clínica-escola de Fonoaudiologia no Rio Grande do Sul.

\section{MÉTODOS}

Foram utilizados 113 prontuários de pacientes com diagnóstico de disfonia organofuncional, na faixa etária de cinco a 76 anos e a média de idade de 32,29 anos, 41 do sexo masculino e 72 do feminino. Este estudo caracterizou-se como estudo de série, retrospectivo e transversal, pela análise de prontuários de pacientes atendidos no Setor de Voz do Serviço de Atendimento Fonoaudiológico (SAF) da Universidade Federal de Santa Maria (UFSM), no período de 1998 a 2002.

Os critérios de inclusão foram: ter assinado o termo de consentimento livre e esclarecido (TCLE), pois a população-alvo, ao iniciar o processo terapêutico na clínica-escola, recebeu os esclarecimentos necessários sobre o funcionamento do serviço e foi convidada a ler e assinar o TCLE (norma 196/96 da Comissão Nacional de Ética em Pesquisa CONEP), autorizando o uso de seus dados para pesquisa, desde que preservados os dados de identidade e privacidade; ter diagnóstico médico de patologia organofuncional e ter classificação fonoaudiológica, por meio de informações da anamnese e do histórico, de disfonia organofuncional 1,4-7,9 (nódulos, pólipos, edema de Reinke, úlcera de contato, granulomas, leucoplasia ou cistos); apresentar queixa de distúrbios alérgicos (rinite, sinusite, alergias a substâncias como perfumes, pólen, dentre outros) ${ }^{6,15,16}$ e ou de distúrbios digestivos (azia, pirose, esofagite, Doença do Refluxo Gastresofágico, dentre outros) ${ }^{10-14}$. Em relação ao cisto vocal, este faz parte das alterações estruturais mínimas da cobertura das pregas vocais, sendo considerado como disfonia funcional por inadaptação vocal anatômica, no entanto, a literatura ${ }^{1}$ deixa claro que o cisto pode ser adquirido frente ao fonotrauma por comportamentos de uso vocal incorreto - portanto, no presente trabalho, foram considerados apenas os casos de cistos vocais organonofuncionais - o que foi verificado pela anamnese fonoaudiológica e pelo histórico do paciente.

Como critério de exclusão, considerou-se a ausência de diagnóstico otorrinolaringológico de patologia organofuncional e ausência de diagnóstico fonoaudiológico de disfonia organofuncional ${ }^{1,4-7,9}$.

Para a coleta de dados deste grupo de estudo, levaram-se em conta as seguintes categorias, obtidas por meio da anamnese e dos laudos médicos e fonoaudiológicos presentes nos prontuários: apresentar queixa de distúrbios alérgicos e o tipo (rinite, bronquite, asma, faringite, alergias a substâncias 
como perfumes, pólen, etc) 6,15,16; apresentar queixa de distúrbios digestivos e o tipo (refluxo gastresofágico, azia, gastrite, úlcera estomacal, e hérnia de hiato) ${ }^{10-14}$; apresentar queixa de ambos, distúrbios alérgicos e digestivos, e os tipos; não apresentar queixas de distúrbios alérgicos ou digestivos; tipo de patologia laríngea diagnosticada e hipótese diagnóstica fonoaudiológica ${ }^{1,4-7,9}$.

A população-alvo, ao iniciar o processo terapêutico na clínica-escola, recebeu os esclarecimentos necessários sobre o funcionamento do serviço e foi convidada a ler e assinar um Termo de Consentimento Livre e Esclarecido (norma 196/96 da Comissão Nacional de Ética em Pesquisa - CONEP), autorizando o uso de seus dados para pesquisa, desde que preservados os dados de identidade $e$ privacidade. O projeto de pesquisa deste estudo foi aprovado pelo Comitê de Ética em Pesquisa da instituição de origem, sob o número 074/2005.

O procedimento estatístico utilizado para análise dos dados foi o Teste de Hipótese para Duas Proporções, realizado no pacote estatístico Primer/87 MSDOS, ao nível de significância ( $\alpha=5 \%$ ), aplicado para testar a significância entre as proporções. $O$ critério de decisão foi de: $\mathrm{H}_{\mathrm{o}}$ : Não significativo (não existe diferença entre as proporções); $\mathrm{H}_{1}$ Significativo (existe diferença entre as proporções). Logo, se $P_{\text {calculado }}$ for maior $P_{\text {tabelado }}=$ Não significativo (NS) e se $\mathrm{P}_{\text {calculado }}$ for menor ou igual $\mathrm{P}_{\text {tabelado }}=$ Significativo (S). $O \mathrm{P}_{\text {tabelado }}$ é considerado 0,05 ou $5 \%$.

\section{RESULTADOS}

Estudou-se a presença ou ausência de queixas de distúrbios alérgicos e/ou digestivos em 113 prontuários de pacientes com diagnóstico de disfonia organofuncional. Nas Figuras 1 e 2, é apresentada a análise estatística dos dados sobre a presença ou ausência de queixas de distúrbios alérgicos e/ou digestivos e sua significância. Na Figura 3, evidencia-se a frequência das queixas de distúrbios alérgicos e ou digestivos e, na Figura 4, ilustram-se as queixas de distúrbios alérgicos e ou digestivos nos pacientes estudados.

Observou-se, com significância estatística, que $53 \%$ (60) tinham queixas de distúrbios alérgicos e/ ou digestivos e $47 \%$ (53) não tinham queixas desses distúrbios (Tabela 1 - Figura 1). Daqueles sujeitos com queixa de algum dos distúrbios, 38\% (43) apresentavam apenas queixa de distúrbio alérgico (significância estatística), 5\% (6) apenas de distúrbio digestivo, e 10\% (11) tinham queixas de ambos os distúrbios (Tabela 1, Figuras 2 e 3).

\section{DISCUSSÃO}

Nos sujeitos com disfonia organofuncional deste trabalho $(n=113)$, pôde-se observar que houve presença de queixas de distúrbios alérgicos e ou digestivos em 60 sujeitos (53\%) (Tabela 1, Figura 1), evidenciando a necessidade da avaliação otorrinolaringológica e da conduta terapêutica do médico, em relação a esse tipo de queixa, favorecendo o tratamento e o prognóstico fonoaudiológico quanto à disfonia ${ }^{5}$.

Com base nos dados da Tabela 1, observou-se que a ocorrência de queixas de distúrbios alérgicos e/ou digestivos em pacientes com disfonia organofuncional foi estatisticamente significante, estando de acordo com os estudos de outros autores ${ }^{6,11}$ que apontam com ênfase a nefasta influência dos mesmos sobre a produção vocal em todos os seus níveis (respiratório, fonatório e articulatório/ressonantal).

Os distúrbios alérgicos, bem como os digestivos, podem interferir diretamente no estabelecimento e prognóstico das disfonias, e existe uma série de outros fatores que podem influenciar a instalação e evolução das mesmas, tais como outros hábitos inadequados que incluem o tabagismo, etilismo, uso de ar condicionado, e o próprio comportamento vocal, ou seja, o fonotrauma devido ao uso incorreto da voz ${ }^{1,5,6}$.

É comum que os pacientes com tendência ao desenvolvimento de hábitos vocais inadequados utilizem diferentes práticas abusivas e a somatória de diferentes usos incorretos da voz tem grande peso na redução da resistência vocal e no estabelecimento de uma disfonia. O grau de resposta e de influência desses fatores sobre a voz varia entre os indivíduos ${ }^{6}$. De qualquer forma, a literatura aponta que um dos grandes grupos de fatores causais das disfonias é composto pelos fatores alérgicos ${ }^{6,17}$, o que converge com os resultados encontrados na presente pesquisa (Tabela 1 - Figura 2).

$\mathrm{Na}$ amostra estudada, pôde-se observar significância estatística das queixas de distúrbios alérgicos em relação àquelas de distúrbios digestivos (Tabela 1 - Figura 2). As alergias são pouco frequentes na maioria dos estados brasileiros, porém apresentam manifestações importantes no sul do Brasil e em algumas regiões montanhosas e frias do sudeste ${ }^{18}$, o que converge com os resultados desta pesquisa. Além disso, as mudanças bruscas de temperatura podem causar uma mudança vascular que pode levar momentaneamente a uma diminuição dos mecanismos de defesa, causando edemas nas mucosas, descarga de muco ou até problemas inflamatórios ${ }^{6}$.

Com base nos achados da Tabela 1, observouse que, no grupo em questão, houve significância 
Tabela 1 - Análise estatística dos dados sobre queixas de distúrbios alérgicos e ou digestivos nos pacientes com disfonia organofuncional e sua significância

\begin{tabular}{|c|c|c|c|c|c|c|}
\hline & Proporção & $n$ & $\%$ & Pcal & Ptab & Hipótese \\
\hline \multirow{6}{*}{$\begin{array}{l}\text { Presença ou ausência de } \\
\text { queixas de distúrbios } \\
\text { alérgicos e/ou digestivos } \\
\text { em sujeitos com disfonia } \\
\text { organofuncional }\end{array}$} & $\begin{array}{l}\text { Presença de queixas de } \\
\text { distúrbios alérgicos e/ou } \\
\text { digestivos }\end{array}$ & 60 & 53 & \multirow[b]{2}{*}{0.006} & \multirow[b]{2}{*}{0.05} & \multirow{2}{*}{$\begin{array}{l}\text { Rejeita-se } \\
\mathrm{H}_{0}^{*}\end{array}$} \\
\hline & $\begin{array}{l}\text { Ausência de queixas de } \\
\text { distúrbios alérgicos e/ou } \\
\text { digestivos }\end{array}$ & 53 & 47 & & & \\
\hline & $\begin{array}{l}\text { Somente queixas de } \\
\text { distúrbios alérgicos }\end{array}$ & 43 & 38 & \multirow[b]{2}{*}{0.507} & \multirow[b]{2}{*}{0.05} & \multirow[b]{2}{*}{$\begin{array}{c}\text { Aceita-se } \\
\mathrm{H}_{0}\end{array}$} \\
\hline & $\begin{array}{l}\text { Sem queixas de distúrbio } \\
\text { alérgico e/ou digestivo }\end{array}$ & 53 & 47 & & & \\
\hline & $\begin{array}{l}\text { Somente queixas de } \\
\text { distúrbios digestivos }\end{array}$ & 6 & 5 & \multirow[b]{2}{*}{0.886} & \multirow[b]{2}{*}{0.05} & \multirow[b]{2}{*}{$\begin{array}{c}\text { Aceita-se } \\
\mathrm{H}_{0}\end{array}$} \\
\hline & $\begin{array}{l}\text { Sem queixas de distúrbio } \\
\text { alérgico e ou digestivo }\end{array}$ & 53 & 47 & & & \\
\hline \multirow{6}{*}{$\begin{array}{l}\text { Ocorrência de queixas de } \\
\text { distúrbios alérgicos e/ou } \\
\text { digestivos em sujeitos com } \\
\text { disfonia organofuncional }\end{array}$} & $\begin{array}{l}\text { Queixas de distúrbio } \\
\text { alérgico }\end{array}$ & 43 & 38 & \multirow{3}{*}{0.013} & \multirow{2}{*}{0.05} & \multirow{2}{*}{$\begin{array}{l}\text { Rejeita-se } \\
\mathrm{H}_{0}^{*}\end{array}$} \\
\hline & $\begin{array}{l}\text { Queixas de distúrbio } \\
\text { digestivo }\end{array}$ & 6 & 5 & & & \\
\hline & $\begin{array}{l}\text { Queixas de distúrbio } \\
\text { alérgico }\end{array}$ & 43 & 38 & & \multirow{3}{*}{0.05} & \multirow[b]{2}{*}{$\begin{array}{c}\text { Rejeita-se } \\
\mathrm{H}_{0}{ }^{*}\end{array}$} \\
\hline & $\begin{array}{l}\text { Queixas de distúrbio } \\
\text { alérgico e distúrbio } \\
\text { digestivo }\end{array}$ & 11 & 10 & \multirow[t]{2}{*}{0.004} & & \\
\hline & $\begin{array}{l}\text { Queixas de distúrbios } \\
\text { digestivos }\end{array}$ & 6 & 5 & & & \multirow[b]{2}{*}{$\begin{array}{c}\text { Aceita-se } \\
\mathrm{H}_{0}\end{array}$} \\
\hline & $\begin{array}{l}\text { Queixas de distúrbios } \\
\text { alérgicos e distúrbios } \\
\text { digestivos }\end{array}$ & 11 & 10 & 0.804 & 0.05 & \\
\hline
\end{tabular}

* Significativo (existe diferença entre as proporções) Teste de Hipótese para Duas Proporções $p=(0,05)$

estatística a favor dos indivíduos com queixas de distúrbios alérgicos e ou digestivos, em relação aos indivíduos sem tais queixas. Alguns autores ${ }^{6}$ afirmam que os distúrbios alérgicos e ou digestivos interferem no estabelecimento e na evolução do tratamento das disfonias. Existe uma série de alterações que podem influenciar o estabelecimento do quadro disfônico, contribuindo para a manutenção ou para a recorrência das crises disfônicas. As principais alterações dizem respeito aos distúrbios alérgicos, faríngeos, bucais, nasais, otológicos, pulmonares, digestivos, hormonais, e neurovegetativos ${ }^{6}$, havendo a necessidade de avaliações detalhadas e cuidadosas, com vistas a não comprometer a efetividade do tratamento e o prognóstico por negligência de algum desses fatores interferentes.

Em determinado estudo ${ }^{19}$, com 451 participantes, sobre a prevalência e fatores de risco da disfonia em professores, os autores observaram que sintomas sugestivos de rinite alérgica foram referidos por $25,7 \%$ professores, já aqueles sugestivos de
Doença do Refluxo Gastresofágico (DRGE) foram referidos por $22,2 \%$. Os autores puderam observar que os sintomas de rinite alérgica se mostraram muito mais comuns em professores com disfonia frequente ou constante, o mesmo pôde ser observado no que diz respeito à DRGE, resultados que vão ao encontro daqueles encontrados na presente pesquisa, na qual as queixas de rinite alérgica e DRGE foram as mais frequentes (Figura 4).

A histamina, liberada durante quadros alérgicos, produz vários efeitos no organismo ${ }^{20}$. Na mucosa nasal, ocorrem diversas reações como vasodilatação e permeabilidade vascular, ocasionando obstrução nasal, aumento da produção de muco, espirros por estimulação das terminações nervosas, o que pode levar às alterações ressonantais vocais e compensações inadequadas por parte do paciente, piorando a disfonia. Podem ocorrer problemas secundários à alergia ${ }^{6}$, tais como usos vocais incorretos, pigarro e tosse, que agridem a mucosa das pregas vocais, favorecendo o aparecimento de lesões 
Presença ou ausência de queixas

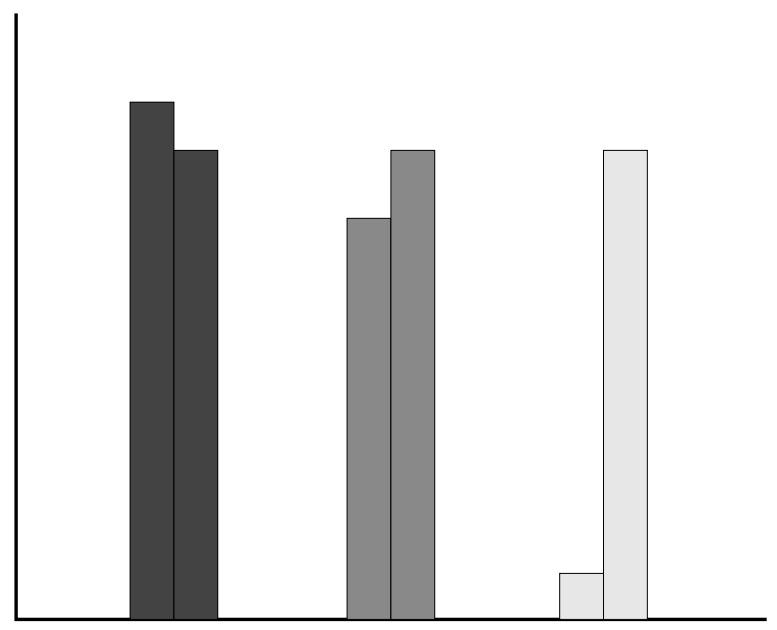

Presença de queixas $n=60(53 \%) X$

ausência de queixas de distúrbios alérgicos

e/ou digestivos $n=53(47 \%)^{*}$

Somente queixa de distúrbios alérgicos

$n=43(38 \%) X$ sem queixas $n=53(47 \%)$

Somente queixa de distúrbios digstivos $\mathrm{n}=6$

$(5 \%) X$ sem queixas $n=53(47 \%)$

* significativo (existe diferença entre as proporções)

Figura 1 - Análise estatística dos dados sobre a presença ou ausência de queixas de distúrbios alérgicos e ou digestivos nos pacientes com disfonia organofuncional e sua significância

organofuncionais. A alergia agride a mucosa, podendo causar espessamento e edema das pregas vocais ${ }^{6}$, dificultando a vibração das mesmas, gerando disfonia em nível laríngeo e levando a compensações inadequadas. Isto está de acordo com o que foi observado, neste estudo, em que uma parcela significativa de indivíduos com disfonia organofuncional tinha queixa de distúrbios alérgicos (Figuras 2 e 3), em especial de rinite alérgica (Figura 4).

A hiponasalidade, disfonia em nível ressonantal, pode estar relacionada com quadros alérgicos que ocasionam respiração oral, levando ao ressecamento do trato vocal ${ }^{21}$. Os malefícios da respiração oral, além do ressecamento do trato vocal ${ }^{21}$, que dificulta a adequada vibração das pregas vocais, gerando esforço, sensações desagradáveis à fonação, e possível fonotrauma, incluem o choque térmico do ar não aquecido pelas fossas nasais, que pode gerar edema e espessamento das pregas vocais, e a não filtragem do ar inspirado, que

\section{Ocorrência de queixas}

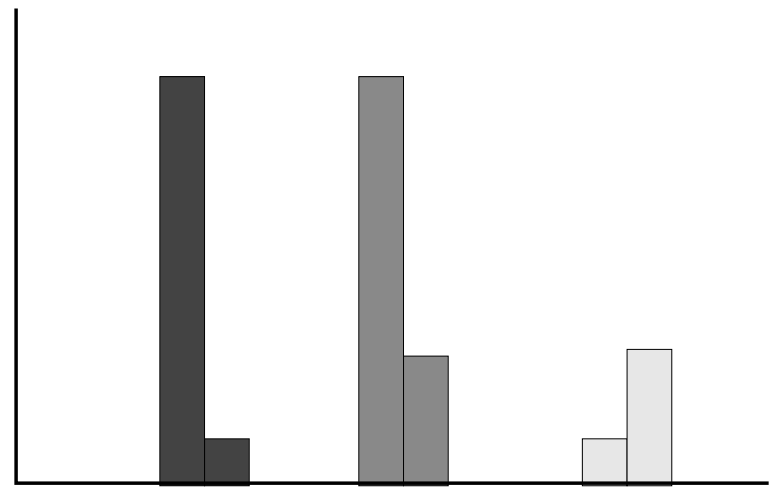

Queixas de distúrbios alérgicas $n=43(38 \%)$

$X$ queixas de distúrbios digestivos $n=6(5 \%)^{*}$

Queixas de distúrbios alérgicos $n=43(38 \%) X$ queixas de distúrbios alérgicos e digestivos $n=11(10 \%)^{*}$

Queixas de distúrbios digestivos $\mathrm{n}=6(5 \%) \mathrm{X}$ queixas de distúrbios alérgicos e digestivos $n=11(10 \%)$

Figura 2 - Análise estatística dos dados sobre a ocorrência de queixas de distúrbios alérgicos e ou digestivos nos pacientes com disfonia organofuncional e sua significância

pode acumular impurezas sobre as mesmas. Todos esses fatos podem, como discutido anteriormente, tornar a vibração em nível glótico alterada e a voz disfônica ${ }^{6,17}$, como o que foi observado no presente estudo, em que foi significativa a queixa de distúrbios alérgicos em indivíduos com disfonia organofuncional (Figuras 2 e 3 ).

Os medicamentos utilizados no tratamento dos quadros alérgicos, como a rinite alérgica, podem causar um efeito de rebote quando, ao terminar o efeito de vasoconstrição da droga, há um retorno do edema e da congestão em grau maior do que o anterior. Tal efeito pode gerar um círculo vicioso e o paciente continuará a tomar o medicamento para amenizar o efeito colateral. A longa duração da vasoconstrição pode resultar na redução do fluxo sanguíneo para a mucosa das pregas vocais, ocasionando perda de equilíbrio de fluido, levando a uma rigidez da mesma e, em consequência, rouquidão. Ainda, pode provocar um efeito sedativo ao mascarar a dor ao falar, o que gera um esforço excessivo à fonação, podendo causar danos à membrana mucosa das pegas vocais ${ }^{22}$, o que 


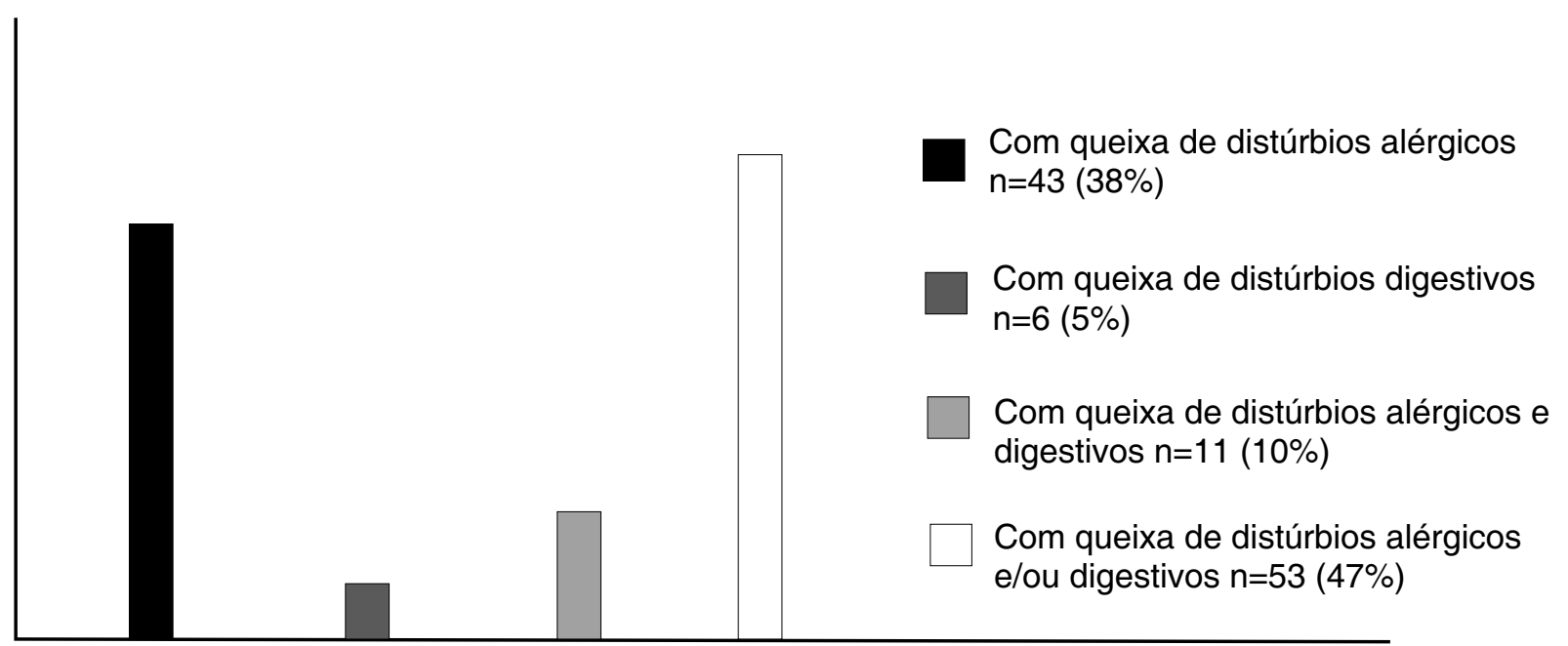

Figura 3 - Frequência das queixas de distúrbios alérgicos e ou digestivos nos pacientes com disfonia organofuncional $(n=113)$

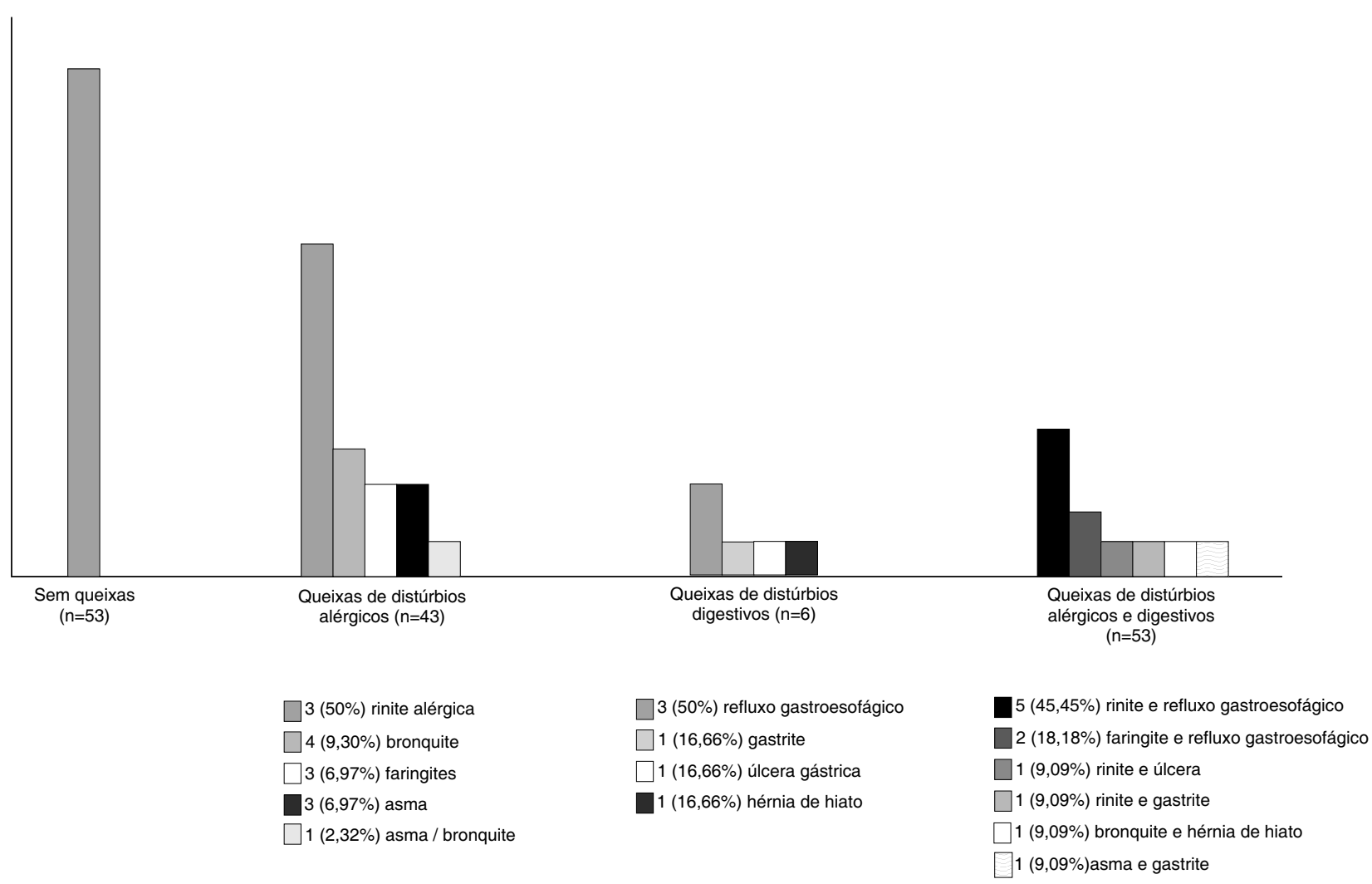

Figura 4-Queixas de distúrbios alérgicos e ou digestivos nos pacientes com disfonia organofuncional $(n=113)$

contribui, desta forma, para o estabelecimento de disfonias organofuncionais (Figuras 3 e 4).

Após a instalação de um quadro alérgico, diversos autores relatam que os pacientes cometem comportamentos agressivos às pregas vocais, como o pigarro e a tosse, com o objetivo de "limpar a garganta", em função da sensação de presença de corpo estranho ou de secreção ${ }^{21}$, também favorecendo o fonotrauma e a possibilidade de aparecimento de lesões organofuncionais nas pregas vocais ${ }^{6}$. Dados que corroboram aqueles encontrados no presente estudo (Figuras 1, 2, 3 e 4). 
No que se refere à correlação do aspecto de nódulos vocais com a patologia nasal, nos nódulos mais recentes (edematosos) a doença nasal mais comum foi a rinite alérgica. $O$ autor verificou ainda que, mesmo após o controle da crise, quando os sintomas nasais desaparecem momentaneamente, os nódulos permanecem. Este fato foi atribuído à agressão da mucosa laríngea provocada pela alergia, tornado-a mais vulnerável a outros agressores como o cigarro, endocrinopatias, usos vocais incorretos, refluxo gastresofágico, e irritantes do ar. O que vai ao encontro da significância estatística de queixas de distúrbios alérgicos em pacientes com disfonia organofuncional no presente estudo (Figuras 2 e 3 ).

No indivíduo alérgico, ocorre aumento da produção de muco mais viscoso, induzindo o sujeito a pigarrear e/ou tossir ${ }^{21}$. As modificações na camada de muco que recobre o epitélio das pregas vocais reduzem o efeito de Bernoulli, prejudicando o ciclo vibratório, gerando alterações na qualidade vocal, ataques vocais bruscos, sensações de ardor, aperto, e pigarro.

A rouquidão aguda deve-se, na maioria dos casos, à inflamação e edema da laringe, relacionadas a uma causa específica, como o uso excessivo da voz, infecção do trato respiratório superior ou irritação do mesmo. Uma das causas primárias da perda de voz é a laringite aguda infecciosa, habitualmente viral. Infecções em outras estruturas do trato respiratório que causem tosse, aumento da produção de muco, necessidade de pigarrear ou de respiração oral, podem também afetar a laringe. A exposição a alérgenos pode também causar o típico edema e eritema visto em laringite infecciosa ou irritativa. Além disso, a irritação aguda da laringe pode ser causada por refluxo gastresofágico (RGE), que provoca uma rouquidão tipicamente matutina, pela inalação de substâncias irritantes, como o consumo de álcool e tabaco ou a própria rinite alérgica ${ }^{11,14}$. $\mathrm{O}$ que vai ao encontro dos resultados encontrados no presente estudo (Figuras 1, 2 e 3).

Diversos autores $7,10,12,14,22,23$ relatam a influência dos distúrbios digestivos na voz. Tais distúrbios (dor e dificuldade à deglutição, engasgo, acidez, refluxo gastresofágico, gastrite, digestão lenta e prisão de ventre, dentre outros) prejudicam o processo de fonação de diversas formas, como ao impedir o livre movimento do diafragma durante a respiração, gerando incoordenação entre o fluxo expiratório e a sonorização glótica, e também dificuldades no controle do abastecimento inspiratório, conforme a demanda fonatória do indivíduo. Alguma dessas queixas, como refluxo gastresofágico e gastrite, foram citadas no presente estudo (Figura 4).

Também é importante salientar a aspiração de secreções e alterações secretórias e vasomotoras por estímulo vagal, que podem ter um papel importante na incidência de laringites, podendo levar a alterações estruturais laríngeas como a paquidermia posterior, os granulomas de terço posterior, úlceras de contato, estenoses laríngeas, carcinoma laríngeo, edema, nódulos e pólipos vocais, espessamentos, irregularidades e sulcos da mucosa das pregas vocais, desordens pulmonares como asma e pneumonia, dentre outros ${ }^{12-14}$. Ou seja, essas alterações estão relacionadas ao estabelecimento de patologias organofuncionais, como foi observado neste estudo (Figuras 3 e 4).

Alguns pesquisadores ${ }^{24}$, ao investigarem a relação entre a Doença do Refluxo Gastresofágico (DRGE), alterações laríngeas, e disfonia, em crianças de 0 a 12 anos de idade com diagnóstico confirmado de DRGE, observaram que a totalidade da amostra apresentou alterações laríngeas, sendo que a maioria apresentava nódulos vocais e disfonia, sem história pregressa de usos incorretos da voz. Verificou-se perfeita correlação estatística entre DRGE e alterações laríngeas e disfonia, na amostra do trabalho, sendo salientado pelas autoras que se deve estar atento, na clínica gastroenterológica, otorrinolaringológica, e fonoaudiológica, a crianças com alterações orgânicas laríngeas e disfonia, pois as mesmas podem fazer parte de um quadro de DRGE. O que vai ao encontro da alta frequência de queixa de DRGE na presente pesquisa (Figura 4).

O refluxo de conteúdo gástrico pode causar, além dos vários efeitos na laringe, alterações na cavidade oral e faríngea como secreção nasal posterior, mau cheiro no nariz e obstrução nasal ${ }^{6}$, aftas e estomatites de repetição, cáries e periodontites, devido a alterações no ph oral, hiper-salivação, halitose, queimação oral, engasgos, disfagia, odinofagia, deglutições repetidas, soluço exagerado, aerofagia, ruminação, ardência e dor na faringe ${ }^{11}$. Ou seja, o refluxo do conteúdo gástrico parece estar diretamente relacionado ao estabelecimento das disfonias organofuncionais (Figura 4).

Algumas das principais queixas encontradas 12-14,22,25 em pacientes com refluxo gastresofágico são disfagia, sensação de corpo estranho na faringe, tosse crônica, e pigarro. A tosse e o pigarro podem apresentar dois fatores relevantes que os desencadeiam: a própria acidez da laringe e a irritação dos terminais nervosos diafragmáticos que causam a tosse reflexa. Em um estudo realizado com 157 indivíduos, as queixas mais frequentes foram: rouquidão $(69,42 \%)$, globus faríngeo $(65,60 \%)$, pigarro $(52,86 \%)$, azia $(33,12 \%)$, tosse $(18,97 \%)$, odinofagia $(11,46 \%)$, disfagia $(10,82 \%)$, e engasgos $(2,54 \%){ }^{11}$. Fatores que podem estar relacionados ao estabelecimento das disfonias organofuncionais (Figura 4). 
Em pesquisa realizada ${ }^{26}$, os sintomas mais comuns do refluxo gastresofágico foram rouquidão, dor de garganta, garganta irritada ${ }^{27}$, e gotejamento nasal posterior, o que também concorda com outros estudos ${ }^{7,14}$. Muitos pacientes com refluxo gastresofágico têm sintomas como rouquidão pela manhã, halitose, dor de "garganta", tosse crônica, asma, dentre outros ${ }^{11}$. O refluxo gastresofágico pode ser a causa primária ou um co-fator significante em quase dois terços de pacientes com desordens vocais $^{7,14}$, o que concorda com os achados do presente estudo (Figura 4).

Menos da metade dos pacientes com DRGE tem sintomas de azia ou regurgitação, portanto, podem não descobrir o refluxo gastresofágico ${ }^{13}$. Alguns autores $5,6,14$ atribuem o insucesso da terapia fonoaudiológica, em alguns casos de nódulos vocais, à presença de refluxo gastresofágico não diagnosticado.

Devem-se realizar mais estudos para esclarecer a prevalência e relações causais entre o refluxo gastresofágico ${ }^{12,13} \mathrm{e}$ as desordens laringofaríngeas e pulmonares em adultos e crianças ${ }^{21}$.

Com base no que foi exposto, é possível que exista relação entre as queixas de distúrbios alérgicos e/ou digestivos e a disfonia organofuncional, uma vez que esses distúrbios são co-fatores para o estabelecimento da disfonia e da lesão laríngea. A alta frequência de queixas de distúrbios alérgicos nos indivíduos com disfonia organofuncional, no presente estudo, pode ter relação com o clima do sul do Brasil ${ }^{6,18}$.

\section{CONCLUSÃO}

Dentre as queixas de distúrbios alérgicos, de distúrbios digestivos, e de ambas associadas, as queixas de distúrbios alérgicos foram significativamente mais frequentes nos pacientes com disfonia organofuncional.

Também foi possível concluir que houve significativa frequência de queixas de distúrbios alérgicos e digestivos associados nos pacientes estudados.

Existe uma relação entre as queixas de distúrbios alérgicos e/ou digestivos e a disfonia, uma vez que esses são co-fatores para o estabelecimento da disfonia e da lesão laríngea. A alta frequência de queixas de distúrbios alérgicos nos indivíduos com disfonia organofuncional, no presente estudo, pode ter relação com o clima do sul do Brasil.

\section{ABSTRACT}

Purpose: to check the occurrence of the complains of allergic and digestive disturbance in patients with organic-functional dysphonia that were attended in Speech and Language Therapist School Clinic. Methods: this study is a sequence, retrospective, transverse study. We have done an analysis of 113 patients' medical records with organic-functional dysphonia that had complains of allergic and/ or digestive disturbance, from 05 to 76 -years-old, 41 male and 72 female. The hypothesis test for 2 proportions was used for the statistic analysis of data. Results: there was a significant relationship of complains on associated allergic and digestive disturbance in patients with organic-functional dysphonia among the complains of allergic disturbance, digestive disturbance, and of both associated, the ones of allergic disturbance were significantly more frequent in patients with Organic-functional dysphonia. Conclusion: there is a relationship among the complains of allergic and/or digestive disturbance and the Organic-functional dysphonia, once that they are co-factors for causing dysphonia and larynx lesions. The high frequency concerning complaints of allergic disturbances in people with organicfunctional dysphonia in this study can be linked with the weather in the south of Brazil.

KEYWORDS: Voice Disorders; Voice; Gastroesophageal Reflux; Rhinitis

\section{REFERÊNCIAS}

1. Behlau M, Azevedo R, Pontes P. Conceito de voz normal e classificação das disfonias. In: Behlau M. Voz: o livro do especialista. vol 1. Rio de Janeiro: Revinter; 2001. p.53-79.

2. Penteado RZ, Pereira IMTB. Avaliação do impacto da voz na qualidade de vida de professores. Rev Soc Bras Fonoaudiol. 2003; 2(2):19-28.
3. Batalla FN, Santos PC, Nieto CS, González BS, Sequeiros G. Evaluación perceptual de la disfonía: correlación con los parámetros acústicos y finalidad. Acta Otorrinolaringol Esp. 2004; 55(6):282-7.

4. Stemple JC, Glaze LE, Klaben BG. Clinical voice pathology: theory and management. San Diego: Singular Publishing Group; 2000.

5. Behlau M, Madazio G, Pontes P. Disfonias organofuncionais. In: Behlau M. Voz: o livro do 
especialista. vol 1. Rio de Janeiro: Revinter; 2001. p.295-329.

6. Pinho SMR. Avaliação e tratamento da voz. In: Pinho SMR. Fundamentos em fonoaudiologia: tratando os distúrbios de voz. Rio de Janeiro: Guanabara Koogan; 2003. p.1-40.

7. Hicks DM, Ours TM, Abelson TI, Vaezi MF, Ritcher JE. The prevalence of hypopharynx findings associated with gastroesophageal reflux in normal volunteers. J Voice. 2002; 16(4):564-79.

8. Fletcher HM, Drinnan MJ, Carding PN. Voice care knowledge among clinicians and people with healthy voices or dysphonia. J Voice. 2007; 21(1):80-91.

9. Petter V, Oliveira PAB de, Fischer PD. Relación entre disfonía referida y potenciales factores de riesgo en el trabajo de profesores de la enseñanza fundamental, Porto Alegre - RS. Salud de los Trabajadores. 2006; 14(2):5-12.

10. Pribuisiene R, Uloza V, Kupcinskas L, Jonaitis L. Perceptual and acoustic characteristics of voice changes in reflux laryngitis patient. J Voice. 2006; 20(1):128-36.

11. Burati DO, Duprat AC, Eckley CA, Costa HO. Doença do refluxo gastroesofágico: análise de 157 pacientes. Rev Bras Otorrinolaringol. 2003; 69(4):458-62.

12. Rouev P, Chakarski I, Doskov D, Dimov G, Staykova E. Laryngopharyngeal symptoms and gastroesophageal reflux disease. J Voice. 2005; 19(3):476-80.

13. Kelchner LN, Horne J, Lee L, Klaben B, Stempe JC, Adam S, et al. Reliability of speech-language pathologist and otolaryngologist ratings of laryngeal signs of reflux in an asymptomatic population using the reflux finding score. J Voice. 2007; 21(1): 92-100.

14. Cesari U, Galli J, Ricciardielo F, Cavaliere M, Galli V. Dysphonia and laryngopharyngeal reflux. Acta Otorhinolaryngol Ital. 2004; 24(1):13-9.

15. Silva MAA. Saúde vocal. In: Pinho SMR. Fundamentos em fonoaudiologia: tratando os distúrbios da voz. Rio de Janeiro: Guanabara Koogan; 2003. p.139-45.
16. Oliveira IB. Avaliação fonoaudiológica da voz: reflexões sobre condutas, com enfoques à voz profissional. In: Ferreira LP, Befi-Lopes DM, Limongi SCO. Tratado de fonoaudiologia. São Paulo: Roca; 2004. p.11-24.

17. Paes C, Vieira J, Leonel T, Cunha DA. O impacto da respiração oral no comportamento vocal. J Bras Fonoaudiol. 2005; 5(23):417-23.

18. Miyake MAM. Atualização no tratamento clínico das rinites alérgicas. In: Stamm AEC, organizador. Rinologia. São Paulo: Revinter; 2000. p.71-4.

19. Fuess VLR, Lorenz MC. Disfonia em professores do ensino municipal: prevalência e fatores de risco. Rev Bras Otorrinolaringol. 2003; 69(6):807-12.

20. Moreira Z. Rinite alérgica. Farmácia Técnica. 2005; 2:1-3.

21. Martins RHG, Trindade SHK. A criança disfônica: diagnóstico, tratamento e evolução clínica. Rev Bras Otorrinolaringol. 2003; 69(6):801-6.

22. Fernandes A, Destailleur D, Villela $P$, Eckley CA. Efeitos diferenciados dos medicamentos para refluxo gastroesofágico na região laringofaríngea. In: Behlau M. Laringologia e voz hoje. Rio de Janeiro: Revinter; 1998.

23. Selby JC, Gilbert HR, Lerman JW. Perceptual and acoustic evaluation of individuals with laryngopharyngeal reflux pre- and post-treatment. J Voice. 2003; 17(4):557-70.

24. Moreira CP, Cielo CA. Doença do refluxo gastroesofágico e alterações vocais na infância. Rev Bras Fonoaudiol. 2004; 9(3):168-78.

25. Belafsky PC, Postma GN, Koufman JA. Validity and reliability of the reflux symptom index (RSI). J Voice. 2002; 16(2):274-7.

26. Rothstein SG. Reflux and vocal disorders in singers with bulimia. J Voice. 1998; 12(1):89-90.

27. Koufman JA, Aviv JE, Casiano RR, Shaw GY. Laryngopharyngeal reflux: position statement of the committeeonspeech, voice, andswallowingdisorders of the American Academy of Otolaryngology-Head and Neck Surgery. Otolaryngol Head Neck Surg. 2002; 127(1):32-5.

RECEBIDO EM: 03/10/2007

ACEITO EM: 02/12/2008

Endereço para correspondência:

Leila Susana Finger

Rua João Neves da Fontoura, 716/404

São Leopoldo - RS

CEP: 93010-050

E-mail: leilafiner@yahoo.com.br 